\title{
Restrictions on Gauge Groups in Noncommutative Gauge Theory
}

\author{
Keizo Matsubara \\ Institute for Theoretical Physics, Uppsala University, Box 803, S-75108, Uppsala, Sweden \\ keizo@teorfys.uu.se \\ (March, 2000)
}

\begin{abstract}
We show that the gauge groups $S U(N), S O(N)$ and $S p(N)$ cannot be realized on a flat noncommutative manifold, while it is possible for $U(N)$.
\end{abstract}

\section{INTRODUCTION}

The Yang-Mills theories naturally arise as low energy limits of the theory of open strings. One can obtain YangMills theories with different gauge groups by studying different D-brane configurations (see e.g. [1]). For instance, if we place $N$ D-branes on top of each other in the flat space, the corresponding open string theory gives rise to the Yang-Mills theory with the gauge group $U(N)$. One can also obtain gauge theories with other gauge groups such as $S O(N)$ and $S p(N)$ by using the orientifold construction. In more detail, one combines the spatial reflection $\sigma \rightarrow \pi-\sigma$ on the string world-sheet with the target space reflection, $X^{\mu} \rightarrow-X^{\mu}, \mu=1, \ldots, k$ and $X^{\mu} \rightarrow X^{\mu}, \mu=(k+1), \ldots, 10$. It is the goal of this note to study which gauge groups can be realized in the presence of the background $B$-field when the brane worldvolume turns into a noncommutative space $[2,3,4]$.

Interaction with the $B$-field introduces an extra term into the Polyakov action of the string [4],

$$
\Delta S=\frac{-i}{2} \int_{\Sigma} B_{\mu \nu} \epsilon^{a b} \partial_{a} X^{\mu} \partial_{b} X^{\nu} .
$$

Here $a, b=1,2$ are world-sheet indices, $\Sigma$ is the worldsheet and $B_{\mu \nu}=-B_{\nu \mu}$ is the $B$-field on the target space. One requires the expression (1) to be invariant with respect to the orientifold reflection. This implies the following transformation rules for components of the $B$-field,

$$
\begin{array}{cl}
B_{\|\| \|} \rightarrow-B_{\|\|} & B_{\perp \perp} \rightarrow-B_{\perp \perp} \\
B_{\| \perp} \rightarrow B_{\| \perp} & B_{\perp \|} \rightarrow B_{\perp \|} .
\end{array}
$$

Here the symbols $\|$ and $\perp$ stand for the target space indices $\mu=1, \ldots, k$ and $\mu=(k+1), \ldots, 10$, respectively. In the orientifold construction we finally let the branes lie on the orientifold. The continuity of the $B$-field implies that the $B$-field on the brane, $B_{\|\|}$, vanishes. Hence, the brane world-volumes are commutative since it is $B_{\|\| \|}$which is responsible for the noncommutativity $[2,3,4]$. This consideration indicates that one should encounter difficulties in the construction of the gauge theories with gauge groups $S O(N)$ and $S p(N)$ on noncommutative spaces. Somewhat surprisingly, we find that the reduction from
$U(N)$ to $S U(N)$ in the framework of noncommutative geometry also fails.

\section{THE CLOSURE OF CLASSICAL LIE ALGEBRAS UNDER THE MOYAL COMMUTATOR}

In the flat case the presence of a constant $B$-field turns the D-branes into noncommutative spaces, with the ordinary pointwise multiplication of functions replaced by the Moyal product,

$$
\begin{aligned}
(X * Y)(x) & =\left.\exp \left(\frac{i}{2} \theta^{i j} \partial_{i}^{x} \partial_{j}^{y}\right) X(x) Y(y)\right|_{x=y}= \\
& =X Y+\frac{i}{2} \theta^{i j} \partial_{i} X \partial_{j} Y+\ldots
\end{aligned}
$$

Here $X$ and $Y$ are functions on the D-brane worldvolume, and $\theta^{i j}$ is a real-valued constant antisymmetric tensor constructed of the metric and $B$-field [3]. The Moyal product naturally extends to $N$ by $N$ matrices, formula (3) still applies. One can also introduce the Moyal commutator by the formula,

$$
[X, Y]_{*}=X * Y-Y * X
$$

In what follows we check whether the matrix Lie algebras of the classical Lie groups $S O(N), U(N), S U(N)$ and $S p(N)$ are closed under the Moyal commutator. We choose to work in the fundamental representation of these Lie algebras.

The Lie algebra of $U(N)$ consists of anti-Hermitean matrices, $\overline{X^{t}}=-X$, where the bar stands for complex conjugation. We first show that this algebra is closed under the Moyal commutator. The key observation is the following property of the Moyal product,

$$
\overline{(X * Y)^{t}}=\overline{Y^{t}} * \overline{X^{t}} .
$$

By using the ordinary rules for the transpose of matrices we get,

$$
\begin{aligned}
& (X * Y)^{t}=Y^{t} X^{t}+ \\
& +\frac{i}{2} \theta^{i j} \partial_{j} Y^{t} \partial_{i} X^{t}-\frac{1}{8} \theta^{i j} \theta^{k l} \partial_{j} \partial_{l} Y^{t} \partial_{i} \partial_{k} X^{t}+\ldots
\end{aligned}
$$


The construction for higher order terms is obvious. Now we apply the complex conjugation and rename the indices of $\theta$ to obtain,

$$
\begin{aligned}
& \overline{(X * Y)^{t}}=\overline{Y^{t} X^{t}}+\frac{i}{2} \theta^{i j} \partial_{i} \overline{Y^{t}} \partial_{j} \overline{X^{t}}- \\
& \frac{1}{8} \theta^{i j} \theta^{k l} \partial_{i} \partial_{k} \overline{Y^{t}} \partial_{j} \partial_{l} \overline{X^{t}}+\ldots=\overline{Y^{t}} * \overline{X^{t}}
\end{aligned}
$$

Taking into account $\overline{X^{t}}=-X$ and $\overline{Y^{t}}=-Y$ yields,

$$
\begin{aligned}
\overline{[X, Y]_{*}^{t}} & =\overline{(X * Y)^{t}}-\overline{Y * X)^{t}}= \\
& =\overline{Y^{t}} * \overline{X^{t}}-\overline{X^{t}} * \overline{Y^{t}}= \\
& =Y * X-X * Y=-[X, Y]_{*}
\end{aligned}
$$

which shows that the algebra $U(N)$ is closed under the Moyal commutator.

We now turn to the algebras of $S O(N), S U(N)$ and $S p(N)$. We first show that for $N=2$ these algebras are not closed with respect to the Moyal commutator.

The counter examples for both $S O(2)$ and $S p(2)$ are given by formulas,

$$
X=\left(\begin{array}{cc}
0 & \alpha \\
-\alpha & 0
\end{array}\right) \quad Y=\left(\begin{array}{cc}
0 & \beta \\
-\beta & 0
\end{array}\right)
$$

and the counterexample for $S U(2)$ is

$$
X=\left(\begin{array}{cc}
i \alpha & 0 \\
0 & -i \alpha
\end{array}\right) Y=\left(\begin{array}{cc}
i \beta & 0 \\
0 & -i \beta
\end{array}\right)
$$

Here $\alpha$ and $\beta$ are coordinates on the manifold chosen so that $\theta^{\alpha \beta} \neq 0$. This can always be done unless $\theta=0$ and the Moyal product coincides with the ordinary multiplication of matrix-valued functions. With $X$ and $Y$ as given above one can easily compute the Moyal commutator since all derivatives of order higher than one vanish. The result for both counter examples is

$$
[X, Y]_{*}=i \theta^{\alpha \beta}\left(\begin{array}{cc}
-1 & 0 \\
0 & -1
\end{array}\right) .
$$

Note that this matrix has a nonvanishing trace. Since the Lie algebras of $S O(2), S U(2)$ and $S p(2)$ consist of traceless matrices, we conclude that they are not closed under the Moyal commutator. This also applies to $S O(N), S U(N)$ and $S p(N)$ for arbitrary $N$ because they contain $S O(2), S U(2)$ and $S p(2)$ as subgroups.

Acknowledgements First of all I would like to thank Anton Alekseev who has been very helpful during the preparation of this note. I would also like to thank Ulf Danielsson, Martin Kruczenski and Mårten Stenmark for helpful and encouraging discussions.

[1] J.Polchinski, TASI Lectures on D-branes, hep-th/9611050

[2] M.R.Douglas and C.Hull, D-branes and the Noncommutative torus. J. High Energy Phys. 2(1998)8, hep-th/9711165

[3] V.Schomerus, D-branes and Deformation Quantization, hep-th/9903205

[4] N.Seiberg and E.Witten, String Theory and Noncommutative Geometry, hep-th/9908142 